\title{
REVERSE MATHEMATICS OF TOPOLOGY DIMENSION, PARACOMPACTNESS, AND SPLITTINGS
}

\author{
SAM SANDERS
}

\begin{abstract}
Reverse Mathematics (RM hereafter) is a program in the foundations of mathematics founded by Friedman and developed extensively by Simpson and others. The aim of RM is to find the minimal axioms needed to prove a theorem of ordinary, i.e. non-set-theoretic, mathematics. As suggested by the title, this paper deals with the study of the topological notions of dimension and paracompactness, inside Kohlenbach's higher-order RM. As to splittings, there are some examples in RM of theorems $A, B, C$ such that $A \leftrightarrow(B \wedge C)$, i.e. $A$ can be split into two independent (fairly natural) parts $B$ and $C$, and the aforementioned topological notions give rise to a number of splittings involving highly natural $A, B, C$. Nonetheless, the higher-order picture is markedly different from the second-one: in terms of comprehension axioms, the proof in higher-order RM of e.g. the paracompactness of the unit interval requires full second-order arithmetic, while the second-order/countable version of paracompactness of the unit interval is provable in the base theory $\mathrm{RCA}_{0}$. We obtain similarly 'exceptional' results for the Urysohn identity, the Lindelof lemma, and partitions of unity. We show that our results exhibit a certain robustness, in that they do not depend on the exact definition of cover, even in the absence of the axiom of choice.
\end{abstract}

\section{INTRODUCTION}

Reverse Mathematics (RM hereafter) is a program in the foundations of mathematics initiated around 1975 by Friedman $([18,19])$ and developed extensively by Simpson ([51]) and others. We refer to [52] for a basic introduction to RM and to [4, 51] for an (updated) overview of RM. We will assume basic familiarity with RM, the associated 'Big Five' systems and the 'RM zoo' ([15]). We do introduce Kohlenbach's higher-order RM in some detail Section 2.1.

Topology studies those properties of space that are invariant under continuous deformations. The modern subject was started by Poincaré's Analysis Situs at the end of 19th century, and rapid breakthroughs were established by Brouwer in a two-year period starting 1910, as discussed in [11, p. 168]. We generally base ourselves on the standard monograph by Munkres ([37]).

Now, the RM of topology has been studied inside the framework of second-order arithmetic in e.g. [38 40. This approach makes heavy use of coding to represent uncountable objects via countable approximations. Hunter develops the higherorder RM of topology in 23, and points out some potential problems with the

School of Mathematics, University of Leeds \& Department of Mathematics, TU Darmstadt, Germany

E-mail address: sasander@me.com.

2010 Mathematics Subject Classification. 03B30, 03D65, 03 F35.

Key words and phrases. reverse mathematics, topology, dimension, paracompactness. 
aforementioned coding practice. Hunter's observations constitute our starting point and motivation: working in higher-order RM, we study the RM of notions like dimension and paracompactness motivated as follows: the former is among the most basic/fundamental notions of topology, while the latter has already been studied in second-order RM, e.g. in the context of metrisation theorems (40,51).

As it turns out, the picture we obtain in higher-order RM is completely different from the well-known picture in second-order RM. For instance, in terms of comprehension axioms, the proof in higher-order RM of the paracompactness of the unit interval requires full second-order arithmetic by Theorem 3.11 while the secondorder/countable version of paracompactness of the unit interval is provable in the

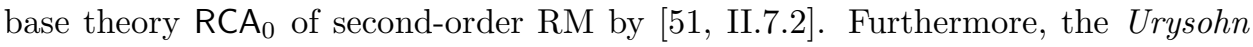
identity connects various notions of dimension, and a proof of this identity for $[0,1]$ similarly requires (comprehension axioms as strong as) full second-order arithmetic. We also study the Lindelöf lemma and partitions of unity.

The aforementioned major difference between second-order and higher-order RM begs the question as to how robust the results in this paper are. For instance, do our theorems depend on the exact definition of cover? What happens if we adopt a more general definition? We show in Sections 3.1, 3.4, and 3.5 that our results indeed boast a lot of robustness, and in particular that they do not depend on the definition of cover, even in the absence of the axiom of (countable) choice. The latter feature is important in view of the topological 'disasters' (see e.g. 24]) that manifest themselves in the absence of the axiom of (countable) choice. A rather elegant base theory is formulated in Section 3.5. based on the neighbourhood function principle from [56.

We also obtain a number of highly natural splittings, where the latter is defined as follows. As discussed in e.g. [22, §6.4], there are (some) theorems $A, B, C$ in the RM zoo such that $A \leftrightarrow(B \wedge C)$, i.e. $A$ can be split into two independent (fairly natural) parts $B$ and $C$ (over $\mathrm{RCA}_{0}$ ). It is fair to say that there are only few natural examples of splittings in second-order RM, though such claims are invariably subjective in nature. A large number of splittings in higher-order RM may be found in 47 .

Finally, like in [42,43], statements of the form 'a proof of this theorem requires full second-order arithmetic' should be interpreted in reference to the usual scale of comprehension axioms that is part of the Gödel hierarchy (see Section 4 for the latter). The previous statement thus (merely) expresses that there is no proof of this theorem using comprehension axioms restricted to a sub-class, like e.g. $\Pi_{k}^{1}$-formulas (with only first and second-order parameters). An intuitive visual clarification may be found in Figure1, where the statement the unit interval is paracompact is shown to be independent of the medium range of the Gödel hierarchy. Similarly, when we say 'provable without the axiom of choice', we ignore the use of the very weak instances of the latter included in the base theory of higher-order RM.

In conclusion, it goes without saying that our results highlight a major difference between second- and higher-order arithmetic, and the associated development of RM. We leave it the reader to draw conclusions from this observation.

\section{Preliminaries}


2.1. Higher-order Reverse Mathematics. We sketch Kohlenbach's higher-order Reverse Mathematics as introduced in [28. In contrast to 'classical' RM, higherorder RM makes use of the much richer language of higher-order arithmetic.

As suggested by its name, higher-order arithmetic extends second-order arithmetic $Z_{2}$. Indeed, while the latter is restricted to numbers and sets of numbers, higher-order arithmetic also has sets of sets of numbers, sets of sets of sets of numbers, et cetera. To formalise this idea, we introduce the collection of all finite types $\mathbf{T}$, defined by the two clauses:

(i) $0 \in \mathbf{T}$ and (ii) If $\sigma, \tau \in \mathbf{T}$ then $(\sigma \rightarrow \tau) \in \mathbf{T}$,

where 0 is the type of natural numbers, and $\sigma \rightarrow \tau$ is the type of mappings from objects of type $\sigma$ to objects of type $\tau$. In this way, $1 \equiv 0 \rightarrow 0$ is the type of functions from numbers to numbers, and where $n+1 \equiv n \rightarrow 0$. We also write $\mathbb{1}$ for the type $1 \rightarrow 1$. Viewing sets as given by characteristic functions, we note that $Z_{2}$ only includes objects of type 0 and 1, i.e. natural numbers and sets thereof.

The language $\mathbf{L}_{\omega}$ includes variables $x^{\rho}, y^{\rho}, z^{\rho}, \ldots$ of any finite type $\rho \in \mathbf{T}$. Types may be omitted when they can be inferred from context. The constants of $\mathrm{L}_{\omega}$ includes the type 0 objects 0,1 and $<_{0},+_{0}, \times_{0},=_{0}$ which are intended to have their usual meaning as operations on $\mathbb{N}$. Equality at higher types is defined in terms of ' $={ }_{0}$ ' as follows: for any objects $x^{\tau}, y^{\tau}$, we have

$$
\left[x={ }_{\tau} y\right] \equiv\left(\forall z_{1}^{\tau_{1}} \ldots z_{k}^{\tau_{k}}\right)\left[x z_{1} \ldots z_{k}={ }_{0} y z_{1} \ldots z_{k}\right]
$$

if the type $\tau$ is composed as $\tau \equiv\left(\tau_{1} \rightarrow \ldots \rightarrow \tau_{k} \rightarrow 0\right)$. Furthermore, $\mathrm{L}_{\omega}$ also includes the recursor constant $\mathbf{R}_{\sigma}$ for any $\sigma \in \mathbf{T}$, which allows for iteration on type $\sigma$-objects as in the special case (2.2). Formulas and terms are defined as usual.

Definition 2.1. The base theory $\mathrm{RCA}_{0}^{\omega}$ consists of the following axioms:

(1) Basic axioms expressing that $0,1,<_{0},+_{0}, \times_{0}$ form an ordered semi-ring with equality $=_{0}$.

(2) Basic axioms defining the well-known $\Pi$ and $\Sigma$ combinators (aka $K$ and $S$ in [2]), which allow for the definition of $\lambda$-abstraction.

(3) The defining axiom of the recursor constant $\mathbf{R}_{0}$ : For $m^{0}$ and $f^{1}$ :

$$
\mathbf{R}_{0}(f, m, 0):=m \text { and } \mathbf{R}_{0}(f, m, n+1):=f\left(n, \mathbf{R}_{0}(f, m, n)\right) .
$$

(4) The axiom of extensionality: for all $\rho, \tau \in \mathbf{T}$, we have:

$$
\left(\forall x^{\rho}, y^{\rho}, \varphi^{\rho \rightarrow \tau}\right)\left[x={ }_{\rho} y \rightarrow \varphi(x)={ }_{\tau} \varphi(y)\right] .
$$

(5) The induction axiom for quantifier-free 1 formulas of $L_{\omega}$.

(6) QF-AC ${ }^{1,0}$ : The quantifier-free axiom of choice as in Definition 2.2

Definition 2.2. The axiom QF-AC consists of the following for all $\sigma, \tau \in \mathbf{T}$ :

$$
\left(\forall x^{\sigma}\right)\left(\exists y^{\tau}\right) A(x, y) \rightarrow\left(\exists Y^{\sigma \rightarrow \tau}\right)\left(\forall x^{\sigma}\right) A(x, Y(x)),
$$

for any quantifier-free formula $A$ in the language of $\mathrm{L}_{\omega}$.

As discussed in [28, §2], $\mathrm{RCA}_{0}^{\omega}$ and $\mathrm{RCA}_{0}$ prove the same sentences 'up to language' as the latter is set-based and the former function-based. Recursion as in (2.2) is called primitive recursion; the class of functionals obtained from $\mathbf{R}_{\rho}$ for all $\rho \in \mathbf{T}$ is called Gödel's system $T$ of all (higher-order) primitive recursive functionals.

\footnotetext{
${ }^{1}$ To be absolutely clear, variables (of any finite type) are allowed in quantifier-free formulas of the language $\mathrm{L}_{\omega}$ : only quantifiers are banned.
} 
We use the usual notations for natural, rational, and real numbers, and the associated functions, as introduced in [28, p. 288-289].

Definition 2.3 (Real numbers and related notions in $\mathrm{RCA}_{0}^{\omega}$ ).

(1) Natural numbers correspond to type zero objects, and we use ' $n^{0}$ ' and ' $n \in \mathbb{N}$ ' interchangeably. Rational numbers are defined as signed quotients of natural numbers, and ' $q \in \mathbb{Q}$ ' and ' $\angle \mathbb{Q}$ ' have their usual meaning.

(2) Real numbers are coded by fast-converging Cauchy sequences $q_{(\cdot)}: \mathbb{N} \rightarrow \mathbb{Q}$, i.e. such that $\left.\left(\forall n^{0}, i^{0}\right)\left(\mid q_{n}-q_{n+i}\right) \mid<\mathbb{Q} \frac{1}{2^{n}}\right)$. We use Kohlenbach's 'hat function' from [28, p. 289] to guarantee that every $q^{1}$ defines a real number.

(3) We write ' $x \in \mathbb{R}$ ' to express that $x^{1}:=\left(q_{(\cdot)}^{1}\right)$ represents a real as in the previous item and write $[x](k):=q_{k}$ for the $k$-th approximation of $x$.

(4) Two reals $x, y$ represented by $q_{(\cdot)}$ and $r_{(\cdot)}$ are equal, denoted $x=\mathbb{R} y$, if $\left(\forall n^{0}\right)\left(\left|q_{n}-r_{n}\right| \leq 2^{-n+1}\right)$. Inequality ' $<\mathbb{R}$ ' is defined similarly. We sometimes omit the subscript ' $\mathbb{R}$ ' if it is clear from context.

(5) Functions $F: \mathbb{R} \rightarrow \mathbb{R}$ are represented by $\Phi^{1 \rightarrow 1}$ mapping equal reals to equal reals, i.e. $(\forall x, y \in \mathbb{R})\left(x=_{\mathbb{R}} y \rightarrow \Phi(x)=_{\mathbb{R}} \Phi(y)\right)$.

(6) The relation ' $x \leq_{\tau} y$ ' is defined as in (2.1) but with ' $\leq_{0}$ ' instead of ' $=_{0}$ '. Binary sequences are denoted ' $f$,,$g^{1} \leq_{1} 1$ ', but also ' $f, g \in C$ ' or ' $f, g \in 2^{\mathbb{N}}$ '.

Finally, we mention the ECF-interpretation, of which the technical definition may be found in [55, p. 138, 2.6]. Intuitively speaking, the ECF-interpretation $[A]_{\mathrm{ECF}}$ of a formula $A \in \mathrm{L}_{\omega}$ is just $A$ with all variables of type two and higher replaced by countable representations of continuous functionals. The ECF-interpretation connects $\mathrm{RCA}_{0}^{\omega}$ and $\mathrm{RCA}_{0}$ (see [28, Prop. 3.1]) in that if $\mathrm{RCA}_{0}^{\omega}$ proves $A$, then $\mathrm{RCA}_{0}$ proves $[A]_{\mathrm{ECF}}$, again 'up to language', as RCA $\mathrm{A}_{0}$ is formulated using sets, and $[A]_{\mathrm{ECF}}$ is formulated using types, namely only using type zero and one objects.

2.2. Some axioms of higher-order arithmetic. We introduce some functionals from [28, 42, 43, which give rise to the higher-order counterparts of $Z_{2}$, and some of the Big Five systems. In each case, the higher-order system is a conservative extension of the second-order system, for a fairly broad formula class.

First of all, $\mathrm{ACA}_{0}$ is readily derived from the following 'Turing jump' functional:

$$
\left(\exists \varphi^{2} \leq_{2} 1\right)\left(\forall f^{1}\right)[(\exists n)(f(n)=0) \leftrightarrow \varphi(f)=0] .
$$

and $\mathrm{ACA}_{0}^{\omega} \equiv \mathrm{RCA}_{0}^{\omega}+\left(\exists^{2}\right)$ proves the same $\Pi_{2}^{1}$-sentences as $\mathrm{ACA}_{0}$ by [46, Theorem 2.2]. This functional is discontinuous at $f={ }_{1} 11 \ldots$, and $\left(\exists^{2}\right)$ is equivalent to the existence of $F: \mathbb{R} \rightarrow \mathbb{R}$ such that $F(x)=1$ if $x>_{\mathbb{R}} 0$, and 0 otherwise $([28, \S 3])$. Secondly, $\Pi_{1}^{1}-\mathrm{CA}_{0}$ is readily derived from the following 'Suslin functional':

$$
\left(\exists S^{2} \leq_{2} 1\right)\left(\forall f^{1}\right)\left[\left(\exists g^{1}\right)\left(\forall x^{0}\right)(f(\bar{g} n)=0) \leftrightarrow S(f)=0\right],
$$

and $\Pi_{1}^{1}-\mathrm{CA}_{0}^{\omega} \equiv \mathrm{RCA}_{0}^{\omega}+\left(S^{2}\right)$ proves the same $\Pi_{3}^{1}$-sentences as $\Pi_{1}^{1}-\mathrm{CA}_{0}$ by 46 , Theorem 2.2]. By definition, the Suslin functional $S^{2}$ can decide whether a $\Sigma_{1}^{1}$-formula (as in the left-hand side of $\left(S^{2}\right)$ ) is true or false. Note that we allow formulas with (type one) function parameters, but not with (higher type) functional parameters. Similarly, let $\left(S_{k}^{2}\right)$ state the existence of a functional $S_{k}^{2}$ that decides $\Pi_{k}^{1}$-formulas (only involving type zero and one parameters). We define $\Pi_{k}^{1}-\mathrm{CA}_{0}^{\omega} \equiv \mathrm{RCA}_{0}^{\omega}+\left(S_{k}^{2}\right)$. Thirdly, full second-order arithmetic $Z_{2}$ is readily derived from $\cup_{k} \Pi_{k}^{1}-C A_{0}^{\omega}$, or from:

$$
\left(\exists E^{3} \leq_{3} 1\right)\left(\forall Y^{2}\right)\left[\left(\exists f^{1}\right) Y(f)=0 \leftrightarrow E(Y)=0\right],
$$


and we define $Z_{2}^{\Omega} \equiv \mathrm{RCA}_{0}^{\omega}+\left(\exists^{3}\right)$ and $\mathbf{Z}_{2}^{\omega} \equiv \cup_{k} \Pi_{k}^{1}-\mathrm{CA}_{0}^{\omega}$; both are conservative over $Z_{2}$ by [23, Cor. 2.6], but see Remark 4.1 . The (unique) functional from $\left(\exists^{3}\right)$ is also called ' $\exists$ ' ', and we will use a similar convention for other functionals.

Fourth, the comprehension for Cantor space functional, introduced in 43, yields a conservative extension of $W_{K} L_{0}$ by [28, Prop. 3.15]:

$$
\left(\exists \kappa_{0}^{3} \leq_{3} 1\right)\left(\forall Y^{2}\right)\left[\kappa_{0}(Y)=0 \leftrightarrow(\exists f \in C)(Y(f)>0)\right] .
$$

Kohlenbach has shown $\left[\left(\exists^{2}\right)+\left(\kappa_{0}^{3}\right)\right] \leftrightarrow\left(\exists^{3}\right)$ over $\mathrm{RCA}_{0}^{\omega}$ by [41, Rem. 6.13].

Fifth, recall that the Heine-Borel theorem (aka Cousin's lemma; see 9 , p. 22]) states the existence of a finite sub-cover for an open cover of a compact space. Now, a functional $\Psi: \mathbb{R} \rightarrow \mathbb{R}^{+}$gives rise to the canonical cover $\cup_{x \in I} I_{x}^{\Psi}$ for $I \equiv[0,1]$, where $I_{x}^{\Psi}$ is the open interval $(x-\Psi(x), x+\Psi(x))$. Hence, the uncountable cover $\cup_{x \in I} I_{x}^{\Psi}$ has a finite sub-cover by the Heine-Borel theorem; in symbols:

$$
\left(\forall \Psi: \mathbb{R} \rightarrow \mathbb{R}^{+}\right)\left(\exists y_{1}, \ldots, y_{k} \in I\right)(\forall x \in I)(\exists i \leq k)\left(x \in I_{y_{i}}^{\Psi}\right) .
$$

There is also the highly similar Lindelöf lemma stating the existence of a countable sub-cover of possibly non-compact spaces. We restrict ourselves to $\mathbb{R}$ as follows.

$$
\left(\forall \Psi: \mathbb{R} \rightarrow \mathbb{R}^{+}\right)\left(\exists \Phi^{0 \rightarrow 1}\right)(\forall x \in \mathbb{R})\left(\exists n^{0}\right)\left(x \in I_{\Phi(n)}^{\Psi}\right),
$$

By the results in $42,43, \mathrm{Z}_{2}^{\Omega}$ proves $\mathrm{HBU}$, but $\Pi_{k}^{1}-\mathrm{CA}_{0}^{\omega}$ cannot (for $k \geq 1$ ). The same holds for LIN, if we add QF-AC ${ }^{0,1}$, while the latter implies $\mathrm{HBU} \leftrightarrow[\mathrm{WKL}+\mathrm{LIN}]$. The importance/naturalness of HBU and LIN is discussed in Section 3.1 .

Finally, since Cantor space (denoted $C$ or $2^{\mathbb{N}}$ ) is homeomorphic to a closed subset of $[0,1]$, the former inherits the same property. In particular, for any $G^{2}$, the corresponding 'canonical cover' of $2^{\mathbb{N}}$ is $\cup_{f \in 2^{\mathbb{N}}}[\bar{f} G(f)]$ where $\left[\sigma^{0^{*}}\right]$ is the set of all binary extensions of $\sigma$. By compactness, there is a finite sequence $\left\langle f_{0}, \ldots, f_{n}\right\rangle$ such that the set of $\cup_{i \leq n}\left[\bar{f}_{i} F\left(f_{i}\right)\right]$ still covers $2^{\mathbb{N}}$. By [42, Theorem 3.3], HBU is equivalent to the same compactness property for $C$, as follows:

$$
\left(\forall G^{2}\right)\left(\exists f_{1}, \ldots, f_{k} \in C\right)(\forall f \in C)(\exists i \leq k)\left(f \in\left[\overline{f_{i}} G\left(f_{i}\right)\right]\right) .
$$

We now introduce the specification $\operatorname{SCF}(\Theta)$ for a functional $\Theta^{2 \rightarrow 1^{*}}$ which computes such a finite sub-cover. We refer to such a functional $\Theta$ as a realiser for the compactness of Cantor space, and simplify its type to ' 3 ' to improve readability.

$$
\left(\forall G^{2}\right)(\forall f \in C)(\exists g \in \Theta(G))(f \in[\bar{g} G(g)]) .
$$

Clearly, there is no unique $\Theta$ as in $\operatorname{SCF}(\Theta)$ (just add more binary sequences to $\Theta(G)$ ); nonetheless, we have in the past referred to any $\Theta$ satisfying $\operatorname{SCF}(\Theta)$ as 'the' special fan functional $\Theta$, and we will continue this abuse of language.

\section{Reverse Mathematics of Topology}

We study the RM of theorems of topology pertaining to the following notions: (topological) dimension and the Urysohn identity (Section 3.2) and paracompactness (Section 3.3). We introduce a suitable notion of cover (Section 3.1) and show (Section 3.4) that our aforementioned results are independent of the definition of cover, without making use of the axiom of choice. We discuss similar results for the Lindelöf lemma and partitions of unity (Section 3.4). We formulate a most elegant base theory in Section 3.5 based on the neighbourhood function principle from [56]. 
3.1. Introduction: topology in higher-order arithmetic. We discuss how higher-order arithmetic can accommodate the central topological notion of cover. In particular, we introduce a generalisation of the notion of cover used in 44, 43. and shall show in Section 3.4 that the new notion yields covering lemmas equivalent to the original, without a need for the axiom of countable choice.

First of all, early covering lemmas, like the Cousin and Lindelöf lemmas, did not make use of the (general) notion of cover. Indeed, Cousin and Lindelöf talk about (uncountable) covers of $\mathbb{R}^{2}$ and $\mathbb{R}^{n}$ as follows (resp. in 1895 and 1903):

we suppose that to each point of $S$ corresponds a circle of non-zero finite radius and with this point as centre ([9, p. 22] )

for every point $\mathrm{P}$, let us construct a sphere $\mathrm{S}_{\mathrm{P}}$ with $\mathrm{P}$ as the centre and a variable radius $\rho_{\mathrm{P}}([30$, p. 698]))

To stay close to the original formulation by Cousin and Lindelöf, we introduced in [42,43] the notion of 'canonical' open covers $\cup_{x \in I} I_{x}^{\Psi}$ of $I \equiv[0,1]$ generated by $\Psi: I \rightarrow \mathbb{R}^{+}$and where $I_{x}^{\Psi} \equiv(x-\Psi(x), x+\Psi(x))$. Unfortunately, such covers always involve points that are covered by arbitrarily many intervals; this property makes such covers unsuitable for e.g. the study of topological dimension, in which the (minimal) number of intervals covering a point is central.

Secondly, the previous observation shows that we have to generalise our notion of canonical cover, and we shall do this by considering $\psi: I \rightarrow \mathbb{R}$. i.e. we allow empty $I_{x}^{\psi}$. In this way, we say that ' $\cup_{x \in I} I_{x}^{\psi}$ covers $[0,1]$ ' if $(\forall x \in I)(\exists y \in I)\left(x \in I_{y}^{\psi}\right)$. This notion of cover gives rise to the following version of the Heine-Borel theorem.

$$
(\forall \psi: I \rightarrow \mathbb{R})\left[I \subset \cup_{x \in I} I_{x}^{\psi} \rightarrow\left(\exists y_{1}, \ldots, y_{k} \in I\right)\left(I \subset \cup_{i \leq k} I_{y_{i}}^{\psi}\right)\right] .
$$

We establish in Section 3.4 that our 'new' notion of cover is quite robust by showing that (i) $\mathrm{HBU} \leftrightarrow \mathrm{HBT}$ over $\mathrm{RCA}_{0}^{\omega}+\mathrm{QF}-\mathrm{AC}^{1,1}$, i.e. the new notion of cover is not a real departure from the old one, and (ii) the previous equivalence can also be proved without the axiom of choice. Item (ii) should be viewed in the light of the topological 'disasters' (see e.g. 24]) that apparently happen in the absence of the axiom of (countable) choice. We also show that any notion of cover definable in $Z_{2}^{\Omega}$ inherits the aforementioned 'nice' properties. Thus, we may conclude that our results boast a lot of robustness, and in particular that they do not depend on the definition of cover, even in the absence of the axiom of (countable) choice.

Finally, we discuss the mathematical naturalness of $\mathrm{HBU}$ and $\left(\exists^{2}\right)$.

Remark 3.1. Dirichlet already discusses the characteristic function of the rationals, which is essentially $\exists^{2}$, around 1829 in [14, while Riemann defines a function with countably many discontinuities via a series in his Habilitationsschrift ([26, p. 115]). Furthermore, the Cousin lemma from [9, p. 22], which is essentially HBU, dates back 2 about 135 years. As shown in [42, $\left(\exists^{2}\right)$ and $\mathrm{HBU}$ are essential for the development of the gauge integral ([3]). This integral was introduced by Denjoy (10]), in a different and more complicated form, around the same time as the Lebesgue integral; the reformulation of Denjoy's integral by Henstock and Kurzweil

\footnotetext{
${ }^{2}$ The collected works of Pincherle contain a footnote by the editors (see [45, p. 67]) which states that the associated Teorema (published in 1882) corresponds to the Heine-Borel theorem. Moreover, Weierstrass proves the Heine-Borel theorem (without explicitly formulating it) in 1880 in [60 p. 204]. A detailed motivation for these claims may be found in [31 p. 96-97].
} 
in Riemann-esque terms (see [3. p. 15]), provides a direct and elegant formalisation of the Feynman path integral ([8, 34, 36]) and financial mathematics ([35, 36]).

3.2. The notion of dimension. The notion of dimension of basic spaces like $[0,1]$ or $\mathbb{R}^{n}$ is intuitively clear to most mathematicians, but finding a formal definition of dimension that does not depend on the topology is a non-trivial problem.

We introduce three notions of dimension: the 'topological' dimension $\operatorname{dim} X$ and the 'small' and 'large' inductive dimensions ind $X$ and Ind $X$. We study the RM properties of the Uryoshn identity (11, p. 272]) which expresses that these dimension are equal for a large class of spaces, including separable metric spaces.

First of all, the covering dimension, later generalised to the topological dimension, goes back to Lebesgue. Indeed, Munkres writes the following:

We shall define, for an arbitrary topological space $X$, a notion of topological dimension. It is the "covering dimension" originally defined by Lebesgue. ([37, p. 305])

The following definition of topological dimension may be found in Munkres' seminal monograph [37, p. 161], and in [1, p. 274], [16, Ex. 1.7.E and Prop. 3.2.2].

Definition 3.2. [Order] A collection $\mathcal{A}$ of subsets of the space $X$ is said to have order $m+1$, if some point of $A$ lies in $m+1$ elements of $\mathcal{A}$, and no point of $X$ lies in more than $m+1$ elements of $A$.

Definition 3.3. [Refinement] Given a collection $\mathcal{A}$ of subsets of $X$, a collection $\mathcal{B}$ is said to refine $\mathcal{A}$, or to be a refinement of $\mathcal{A}$ if for each element $B \in \mathcal{B}$ there is an element $A \in \mathcal{A}$ such that $A \subset B$.

Definition 3.4. [Topological dimension] A space $X$ is said to be finite-dimensional if there is $m \in \mathbb{N}$ such that for every open covering $\mathcal{A}$ of $X$, there is an open covering $\mathcal{B}$ of $X$ that refines $\mathcal{A}$ and has order at most $m+1$. The topological dimension of $X$ is the smallest value of $m$ for which this statement holds; we denote it by $\operatorname{dim} X$.

In the context of $\operatorname{RCA}_{0}^{\omega}$, we say that ' $\phi: I \rightarrow \mathbb{R}$ is a refinement of $\psi: I \rightarrow \mathbb{R}$ ' if $(\forall x \in I)(\exists y \in I)\left(I_{x}^{\phi} \subseteq I_{y}^{\psi}\right)$. With this definition in place, statements like 'the topological dimension of $[0,1]$ is at most 1 ', denoted ' $\operatorname{dim}([0,1]) \leq 1$ ', makes perfect sense in $\mathrm{RCA}_{0}^{\omega}$. Such a statement turns out to be quite hard to prove, as full second-order arithmetic is needed to prove HBT by Theorem 3.15

Theorem 3.5. The system $\mathrm{ACA}_{0}^{\omega}+\mathrm{QF}-\mathrm{AC}^{1,1}+[\operatorname{dim}([0,1]) \leq 1]$ proves $\mathrm{HBT}$.

Proof. Let $\psi: I \rightarrow \mathbb{R}$ be such that $\cup_{x \in I} I_{x}^{\psi}$ covers $[0,1]$, and let $\phi: I \rightarrow \mathbb{R}$ be the associated refinement of order at most 1 . Since the innermost formula is $\Sigma_{1}^{0}$ (with parameters), we may apply QF-AC ${ }^{1,1}$ to $(\forall x \in I)(\exists y \in I)\left(x \in I_{y}^{\phi}\right)$ to obtain $\Xi^{1 \rightarrow 1}$ such that $\Xi(x)$ provides such $y$. Define $\zeta^{0 \rightarrow 1}$ as follows: $\zeta(0):=\Xi(0)+\phi(\Xi(0))$ and $\zeta(n+1):=\Xi(\zeta(n))+\phi(\Xi(\zeta(n)))$. Now consider the following formula:

$$
(\exists x \in I)(\forall n \in \mathbb{N})(\zeta(n)<\mathbb{R} x) .
$$

If (3.1) is false, take $x=1$ and note that if $\zeta\left(n_{0}\right) \geq_{\mathbb{R}} 1$, the finite sequence $I_{\Xi(0)}^{\phi}, I_{\Xi(\zeta(0))}^{\phi}, I_{\Xi(\zeta(1))}^{\phi}, \ldots, I_{\Xi\left(\zeta\left(n_{0}+1\right)\right)}^{\phi}$ yield a finite sub-cover of $\cup_{x \in I} I_{x}^{\phi}$. In this case, we apply QF-AC ${ }^{1,1}$ (using also $\left.\left(\exists^{2}\right)\right)$ to $(\forall x \in I)(\exists y \in I)\left(I_{x}^{\phi} \subseteq I_{y}^{\psi}\right)$ to go from a finite sub-cover of $\cup_{x \in I} I_{x}^{\phi}$ to a finite sub-cover of $\cup_{x \in I} I_{x}^{\psi}$, and HBT follows. 
If (3.1) is true, let $x_{0} \in I$ be the least $x \in I$ such that $\varphi(x) \equiv(\forall n \in \mathbb{N})(\zeta(n)<\mathbb{R}$ $x)$. Since $\varphi(x)$ is $\Pi_{1}^{0}$, we can use $\exists^{2}$ and the usual interval-halving technique to find $x_{0}$; alternatively, use the monotone convergence theorem ([51, III.2.2]), provable in $\mathrm{ACA}_{0}$. However, $I_{\Xi\left(x_{0}\right)}^{\phi}$ covers $x_{0}$, and thus for $n_{1}$ large enough, $\zeta(n)$ for $n \geq n_{1}$ will all be in the former interval, by the leastness of $x_{0}$. But then there are points of order 3 in the (by definition non-empty) intersection of $I_{\Xi\left(\zeta\left(n_{1}\right)\right)}^{\phi}$ and $I_{\Xi\left(\zeta\left(n_{1}+1\right)\right)}^{\phi}$, as this intersection is also inside $I_{\Xi\left(x_{0}\right)}^{\phi}$. This observation contradicts the assumption $\operatorname{dim}([0,1]) \leq 1$, and hence (3.1) must be false, and we are done.

The previous theorem has a number of corollaries. First of all, we obtain an equivalence over a weak base theory; we believe the components of the left-hand side to be independent 3 , i.e. that a proper 'splitting' of HBT is achieved.

Corollary 3.6. $\mathrm{RCA}_{0}^{\omega}+\mathrm{QF}-\mathrm{AC}^{1,1}$ proves that $(\mathrm{WKL}+[\operatorname{dim}(I)=1]) \leftrightarrow \mathrm{HBT}$.

Proof. For the forward direction, in case $\left(\exists^{2}\right)$, the proof of the theorem goes through. In case $\neg\left(\exists^{2}\right)$, all $F: \mathbb{R} \rightarrow \mathbb{R}$ are continuous, while all $F^{2}$ are continuous on Baire space, and hence uniformly continuous (and thus bounded) on Cantor space by WKL (see [28, Prop. 3.7 and 3.12] and [29, Prop. 4.10]). Now consider the following statement, which (only) holds since $\psi: I \rightarrow \mathbb{R}$ is continuous:

$$
(\forall f \in C)(\exists q \in I \cap \mathbb{Q})(\exists n \in \mathbb{N}) \underline{\left(\llbracket(f) \in I_{q}^{\psi} \wedge \psi(q) \geq \frac{1}{2^{n}}\right),}
$$

where $r(f)$ is $\sum_{n=0}^{\infty} \frac{f(n)}{2^{n}}$ for binary $f$, and where the underlined formula is $\Sigma_{1}^{0}$. Applying QF-AC ${ }^{1,0}$ to (3.2), there is $\Xi^{2}$ such that $n \leq \Xi(f)$ in (3.2). Since $\Xi$ is bounded on $C$, there is $N_{0} \in \mathbb{N}$ such that

$$
(\forall f \in C)(\exists q \in I \cap \mathbb{Q})\left(\curvearrowleft(f) \in I_{q}^{\psi} \wedge \psi(q) \geq \frac{1}{2^{N_{0}}}\right),
$$

which immediately implies that $\cup_{x \in I} I_{x}^{\psi}$ has a finite sub-cover (generated by rationals), and the latter may be found by applying QF-AC ${ }^{1,0}$ to (3.3) and iterating the choice function at most $2^{N_{0}+1}$ times. Since $\frac{i}{2^{n}}$ has an obvious binary representation, we do not need to convert arbitrary $x \in I$ to binary. We obtain HBT in each case, and $\left(\exists^{2}\right) \vee \neg\left(\exists^{2}\right)$ finishes this direction of the proof.

For the reverse direction, note that $\mathrm{HBT} \rightarrow \mathrm{HBU} \rightarrow \mathrm{WKL}$. To prove $\operatorname{dim}(I)=1$, the finite sub-cover provided by HBT is readily converted to a refinement of order 1 using $\exists^{2}$, as the latter functional can decide equality between real numbers. Now, in case $\neg\left(\exists^{2}\right)$, obtain (3.3) in the same way as above, and let $\Xi$ be a choice function that provides $\Xi(f)=q$. Define $\zeta$ as follows: $\zeta(0):=\Xi(00 \ldots)+\frac{1}{2^{N_{0}}}$ and $\zeta(n+1):=$ $\Xi(\zeta(n))+\frac{1}{2^{N_{0}}}$. For $n>2^{N_{0}+1}$, this function readily yields a finite open cover of $I$ that is also a refinement of the cover generated by $\psi$. Since all points are rationals, we can refine this cover to have order 1 , and $\left(\exists^{2}\right) \vee \neg\left(\exists^{2}\right)$ finishes the proof.

For future reference, we note that the proof also establishes $\operatorname{RCA}_{0}^{\omega}+\neg\left(\exists^{2}\right)+$ WKL $\vdash \mathrm{HBT}$, i.e. the axiom of choice is not used.

It is a natural question (posed before by Hirschfeldt; see [33, §6.1]) whether the axiom of choice is really necessary in the previous (and below) theorems. We answer this question in the negative in Section 3.4.

\footnotetext{
${ }^{3}$ Firstly, $\mathrm{Z}_{2}^{\Omega}+\mathrm{QF}^{-A C}{ }^{0,1}$ does not prove HBU (42,43]). Secondly, $\operatorname{dim}(I)=1$ seems consistent with recursive mathematics by [4. Theorem 6.1, p. 69], i.e. the former cannot imply WKL.
} 
Next, in order to prove the next corollary concerning Urysohn's identity, we introduce the notion of inductive definition as in [16, §1.1.1].

Definition 3.7. [Inductive dimension] We inductively define the small inductive dimension ind $X$ for a topological space $X$ as follows.

(d1) For the empty set $\emptyset$, we define ind $\emptyset=$ Ind $\emptyset=-1$;

(d2) ind $X \leq n$, where $n=0,1, \ldots$, if for every point $x \in X$ and each neighbourhood $V \subset X$ of the point $x$ there exists an open set $U \subset X$ such that $x \in U \subset V$ and ind $(\partial U)<n-1$

(d3) ind $X=n$ if ind $X \leq n$ and ind $X>n-1$, i.e., the inequality ind $X<n-1$ does not hold;

(d4) ind $X=\infty$ if ind $X>n$ for $n=-1,0,1, \ldots$

The large inductive dimension Ind $X$ is obtained by replacing (d2) by:

$\left(\mathrm{d} 2^{*}\right)$ Ind $X<n$, where $n=0,1, \ldots$, if for every closed set $A \subset X$ and each open set $V \subset X$ which contains the set $A$ there exists an open set $U \subset X$ such that $A \subset U \subset V$ and Ind $(\partial U)<n-1$.

If $X$ is Euclidean space, $V$ is generally chosen to be a ball centred at $x$.

In light of Definition 3.7, the (small and large) inductive dimension of singletons of real numbers, or the unit interval, makes sense in $\mathrm{RCA}_{0}^{\omega}$, and is respectively 0 and 1. Moreover, the Urysohn identity is the statement that $\operatorname{dim} X=\operatorname{ind} X=\operatorname{Ind} X$, and holds for a large class of spaces $X$; this identity constitutes one of the main problems in dimension theory, according to [1, p. 274], while it is called the the fundamental theorem of dimension theory in [16].

Corollary 3.8. The system $\mathrm{RCA}_{0}^{\omega}+\mathrm{QF}-\mathrm{AC}^{1,1}$ proves that $\mathrm{HBT}$ is equivalent to: the conjunction of $\mathrm{WKL}$ and Urysohn's identity for the unit interval.

Proof. Immediate from Corollary 3.6 .

3.3. Paracompactness. The notion of paracompactness was introduced in 1944 by Dieudonné in [12] and plays an important role in the characterisation of metrisable spaces via e.g. Smirnov's metrisation theorem (37, p. 261]). The fact that every metric space is paracompact is Stone's theorem (see [21,53] and [37, p. 252]).

Our interest in paracompactness stems in part from its occurrence in classical RM (see e.g. 39, 40, 51]), as detailed in Remark 3.13. The aim of this section is to show that there is a huge difference in logical and computational hardness between the 'second-order/countable' version of paracompactness, and the 'actual' definition. Indeed, the fact that the unit interval is paracompact implies HBT; moreover, the latter can be 'split' into the former plus WKL by Corollary 3.12

Munkres states the following definition of paracompactness in [37, p. 253].

Definition 3.9. [Locally finite] A collection $\mathcal{A}$ of subsets of a space $X$ is locally finite if any $x \in X$ has a neighbourhood that intersects only finitely many $A \in \mathcal{A}$.

Definition 3.10. [Paracompact] A space $X$ is paracompact if every open covering $\mathcal{A}$ of $X$ has a locally finite open refinement $\mathcal{B}$ that covers $X$.

With these definitions, the statement that the unit interval is paracompact, makes sense in $\mathrm{RCA}_{0}^{\omega}$. By Stone's theorem, a metric space is paracompact, but this fact is not provable in ZF alone (see [21]). Similarly, Stone's theorem for the unit 
interval is not provable in any system $\Pi_{k}^{1}-\mathrm{CA}_{0}^{\omega}$ by the following theorem. Note that the results in Section 3.4 yield a proof in $Z_{2}^{\Omega}$ of the paracompactness of $[0,1]$.

Theorem 3.11. The system $\mathrm{ACA}_{0}^{\omega}+\mathrm{QF}-\mathrm{AC}^{1,1}+{ }^{\cdot}[0,1]$ is paracompact' proves $\mathrm{HBT}$.

Proof. We use the proof of Theorem 3.5 with minor modification. Let $\psi: I \rightarrow \mathbb{R}$ be such that $\cup_{x \in I} I_{x}^{\psi}$ covers $[0,1]$, and let $\phi: I \rightarrow \mathbb{R}$ be a locally finite refinement. Assume (3.1), where let $x_{0} \in I, \zeta^{0 \rightarrow 1}, \Xi^{1,1}$ are as in the aforementioned proof. Clearly, any neighbourhood of $x_{0}$ will contain all intervals $I_{\Xi(\zeta(n))}^{\phi}$ for $n$ large enough. This observation contradicts the assumption that $[0,1]$ is paracompact, and hence (3.1) must be false, implying HBT as in the proof of Theorem 3.5 .

The following corollary is proved in the same way as Corollary 3.6 , the lefthand side constitutes a proper 'splitting' of HBT, as the ECF-translation of '[0, 1$]$ is paracompact' is essentially the statement that $[0,1]$ is countably paracompact, and the latter is provable in $\mathrm{RCA}_{0}$ by [51, II.7.2].

Corollary 3.12. $\mathrm{RCA}_{0}^{\omega}+\mathrm{QF}-\mathrm{AC}^{1,1}$ proves $\left[\mathrm{WKL}+{ }^{\prime}[0,1]\right.$ is paracompact'] $\leftrightarrow$ HBT.

Another interpretation of the previous corollary is as follows: by the results in 58, the notion of compactness is equivalent to 'paracompact plus pseudo-compact' for a large class of spaces, and pseudo-compactness essentially expresses that continuous functions are bounded on the space at hand, i.e. the pseudo-compactness of $[0,1]$ is equivalent to WKL by [51, IV.2.3] and [29, Prop. 4.10].

The following remark highlights the difference between 'actual' and 'secondorder/countable' paracompactness. It also suggests formulating Corollary 3.14.

Remark 3.13 (Paracompactness in second-order RM). Simpson proves in 51, II.7.2 that over $\mathrm{RCA}_{0}$, complete separable metric spaces are countably paracompact4, and Mummert in [40, Lemma 4.11] defines a realiser for paracompactness as in [51, II.7.2] inside $\mathrm{ACA}_{0}$. This realiser plays a crucial role in the proof of Mummert's metrisation theorem, called 'MFMT', inside $\Pi_{2}^{1}-\mathrm{CA}_{0}$ (see [40, §4]). Note that $\Pi_{2}^{1}-\mathrm{CA}_{0}$ occurs elsewhere in the RM of topology ([38, 39]). By Theorem 3.11, the (higher-order) statement the unit interval is paracompact is equivalent to HBT, and hence not provable in $\cup_{k} \Pi_{k}^{1}-\mathrm{CA}_{0}^{\omega}$, i.e. there is a huge difference in strength between 'second-order/countable' and 'actual' paracompactness. In fact, the logical hardness of the aforementioned statement dwarfs $\Pi_{2}^{1}-\mathrm{CA}_{0}$ from the RM of topology.

Let us call $\Omega^{\mathbb{1} \rightarrow \mathbb{1}}$ a 'realiser for the paracompactness of [0,1]' if $\Omega(\psi)(1): I \rightarrow \mathbb{R}$ yields a locally finite open refinement of the cover associated to $\psi: I \rightarrow \mathbb{R}$, and if

$$
(\forall x \in I)\left(I_{x}^{\Omega(\psi)(1)} \subseteq I_{\Omega(\psi)(2)(x)}^{\psi}\right),
$$

i.e. the refining cover is 'effectively' included in the original one, just like in [40,51].

Corollary 3.14. A realiser $\Omega^{\mathbb{1} \rightarrow \mathbb{1}}$ for the paracompactness of $[0,1]$, together with Feferman's $\mu$, computes $\Theta$ such that $\operatorname{SCF}(\Theta)$ via a term of Gödel's T.

Proof. Immediate from the proof of Theorems 3.5 and 3.11. Note that $\Xi$ is the identity function in case we consider covers generated by $\Psi: I \rightarrow \mathbb{R}^{+}$as in HBU. Furthermore, a realiser for $\mathrm{HBU}$ computes a realiser for $\mathrm{HBU}_{c}$, i.e. the special fan functional, via a term in Gödel's $T$, as discussed in [42, §3.1]

\footnotetext{
${ }^{4}$ The notion of 'countably paracompact' is well-known from Dowker's theorem (see e.g. 25, p. 172]), but Simpson and Mummert do not use the qualifier 'countable' in 40,51.
} 
As it turns out, the condition (3.4) for a realiser for paracompactness has already been considered, namely as follows.

all proofs of Stone's Theorem (known to the authors) actually prove a stronger conclusion which implies AC. It is based on an idea from $[\ldots]$. Let us call a refinement $\mathcal{V}$ of $\mathcal{U}$ effective if there is a function $a: \mathcal{V} \rightarrow \mathcal{U}$ such that $V \subset a(V)$ for all $V \in \mathcal{V}$. ([21, p. 1217])

As it turns out, the notion of 'effectively paracompact' is intimately connected to the Lindelöf lemma, as discussed in Section 3.4.3.

3.4. Covers in higher-order arithmetic. In Section 3.1, we introduced a generalisation of the notion of cover used in 42,43, while we used this notion in Sections 3.2 and 3.3 to obtain RM results. In this section, we show that these RM results have some robustness: we show that the new notion of cover yields covering lemmas equivalent to the original ones (with the definition from [42,43]), even in the absence of the axiom of choice. We also show that any notion of cover definable in second-order arithmetic inherits these 'nice' properties. We treat the Heine-Borel theorem, the Lindelöf lemma, as well as theorems pertaining to partitions of unity.

3.4.1. The Heine-Borel theorem. We prove HBT $\leftrightarrow \mathrm{HBU}$ with and without the axiom of choice in the base theory. In this way, we observe that our new notion of cover does not really change the Heine-Borel theorem.

Theorem 3.15. The system $\mathrm{RCA}_{0}^{\omega}+\mathrm{QF}-\mathrm{AC}^{1,1}$ proves $\mathrm{HBU} \leftrightarrow \mathrm{HBT}$.

Proof. The reverse direction is immediate. For the forward direction, in case $\neg\left(\exists^{2}\right)$, we obtain $\mathrm{HBU} \rightarrow \mathrm{WKL}$ and proceed as in the proof of Corollary 3.6. In case $\left(\exists^{2}\right)$, let $\psi$ be as in HBT and consider $(\forall x \in I)(\exists y \in I)\left(x \in I_{y}^{\psi}\right)$. Since the innermost formula is $\Sigma_{1}^{0}$, we may apply QF-AC ${ }^{1,1}$ to obtain $\Xi$ such that $(\forall x \in I)\left(x \in I_{\Xi(x)}^{\psi}\right)$. Since $\exists^{2}$ provides a functional that converts real numbers in $I$ to a unique binary representation, we may assume that $\Xi$ is extensional on the reals. Now define $\Psi: I \rightarrow \mathbb{R}^{+}$by $\Psi(x):=\min (|x-(\Xi(x)-\psi(\Xi(x)))|,|x-(\Xi(x)+\psi(\Xi(x)))|)$, and note that $I_{x}^{\Psi} \subseteq I_{\Xi(x)}^{\psi}$. Applying $\mathrm{HBU}$, we obtain a finite sub-cover of $\cup_{x \in I} I_{x}^{\Psi}$, say generated by $y_{1}, \ldots, y_{k} \in I$, and $\cup_{i \leq k} I_{\Xi\left(y_{i}\right)}^{\psi}$ is then a finite sub-cover of $\cup_{x \in I} I_{x}^{\psi}$.

Recall that $\mathrm{HBU}$ is provable in $\mathrm{Z}_{2}^{\Omega}$ by [43, §4], i.e. without the axiom of choice. While the use of QF-AC ${ }^{1,1}$ in HBU $\rightarrow$ HBT seems essential, it is in fact not, by the following theorem. Note that IND is the induction axiom for all formulas in the language of $\mathrm{RCA}_{0}^{\omega}$; the base theory is not stronger than Peano arithmetic.

Theorem 3.16. The system $\mathrm{RCA}_{0}^{\omega}+\mathrm{IND}+\left(\kappa_{0}^{3}\right)$ proves $\mathrm{HBU} \leftrightarrow \mathrm{HBT}$

Proof. The reverse direction is immediate. For the forward direction, in case $\neg\left(\exists^{2}\right)$, we obtain HBU $\rightarrow$ WKL and proceed as in the proof of Corollary [3.6. In case of $\left(\exists^{2}\right)$, let $\psi$ be as in HBT and note that $(\forall x \in I)(\exists y \in I)\left(x \in I_{y}^{\psi}\right)$ implies:

$$
(\forall x \in I)(\exists n \in \mathbb{N}) \underline{(\exists y \in I)\left(\left(x-\frac{1}{2^{n}}, x+\frac{1}{2^{n}}\right) \subseteq I_{y}^{\psi}\right)},
$$

where the underlined formula is decidable thanks to $\left(\exists^{3}\right) \equiv\left[\left(\exists^{2}\right)+\left(\kappa_{0}^{3}\right)\right]$. Hence, applying QF-AC ${ }^{1,0}$ to (3.5), we obtain $\Psi: I \rightarrow \mathbb{R}^{+}$such that $\cup_{x \in I} I_{x}^{\Psi}$ is a canonical 
cover of $I$. Applying $\mathrm{HBU}$, we obtain a finite sub-cover of $\cup_{x \in I} I_{x}^{\Psi}$, say generated by $x_{1}, \ldots, x_{k} \in I$. By definition, we have $(\forall x \in I)(\exists y \in I)\left(I_{x}^{\Psi} \subseteq I_{y}^{\psi}\right)$, and

$$
\left(\forall w^{1^{*}}\right)\left(\exists v^{1^{*}}\right)(\forall i<|w|)\left(I_{w(i)}^{\Psi} \subseteq I_{v(i)}^{\psi}\right)
$$

follows from IND by induction on $|w|$. Applying (3.6) for $w=\left\langle x_{1}, \ldots, x_{k}\right\rangle$, we obtain a finite sub-cover for $\cup_{x \in I} I_{x}^{\psi}$. The law of excluded middle finishes the proof.

As to open questions, we do not know if the base theory proves HBT outright or not. Similarly, we do not know if $\mathrm{RCA}_{0}^{\omega}+\left(\kappa_{0}^{3}\right)$ proves $\mathrm{WKL}$ or not.

In conclusion, we mention two important observations that stem from the above.

First of all, it is easy to see that the first two proofs go through for the HeineBorel theorem for $[0,1]$ based on any 'reasonable' notion of cover. Indeed, as long as the formulas ' $x \in U_{y}$ ' and ' $[a, b] \subseteq U_{x}$ ' for the new notion of cover $\cup_{x \in I} U_{x}$ of $I$ are decidable in $Z_{2}^{\Omega}$, the above proofs go through (assuming $\left(\kappa_{0}^{3}\right)$ ). Since $Z_{2}^{\Omega}$ can decide if $Y: \mathbb{R} \rightarrow\{0,1\}$ represents an open subset of $\mathbb{R}$ (using the textbook definition of open set), this notion of 'reasonable' seems quite reasonable.

Secondly, emulating the proof of Theorem 3.16, we observe that the above results go through in the base theory with $\left(\kappa_{0}^{3}\right)+$ IND instead of QF-AC ${ }^{1,1}$. These include Theorem 3.5. Corollary 3.6, Corollary 3.8. Theorem 3.11, and Corollary 3.12, Thus, these results do not require the axiom of choice in the base theory.

3.4.2. The Lindelöf lemma. We show that the Lindelöf lemma does not depend on the definition of cover, similar to the case of the Heine-Borel theorem. On one hand, since [LIN + WKL] $\leftrightarrow \mathrm{HBU}$, one expects such results. On the other hand, as shown in [43, §5], the strength of the Lindelöf lemma is highly dependent on the exact 5 formulation, but this dependence is not problematic for our context.

We introduce the notion of cover used in [43, §5], as follows. We consider $\psi: I \rightarrow$ $\mathbb{R}^{2}$ and covers $\cup_{x \in I} J_{x}^{\psi}$ in which the interval $J_{x}^{\psi}:=(\psi(x)(1), \psi(x)(2))$ is potentially empty but $(\forall x \in I)(\exists y \in I)\left(x \in J_{y}^{\psi}\right)$. This notion of cover yields a 'strong' version of the Lindelöf lemma, as follows.

$$
\left(\forall \psi: \mathbb{R} \rightarrow \mathbb{R}^{2}\right)\left[\mathbb{R} \subseteq \cup_{x \in \mathbb{R}} J_{x}^{\psi} \rightarrow(\exists f: \mathbb{N} \rightarrow \mathbb{R})\left(\mathbb{R} \subseteq \cup_{n \in \mathbb{N}} J_{f(n)}^{\psi}\right)\right] .
$$

Similar to the proof of [42, Theorem 3.13], one proves that HBT $\leftrightarrow$ [WKL + LIL] over $\mathrm{RCA}_{0}^{\omega}+\mathrm{QF}-\mathrm{AC}^{1,1}$. We first prove that the Lindelöf lemma LIL is equivalent to LIN from [42, §3]. We believe that LIN does not imply countable choice QF-AC ${ }^{0,1}$.

Theorem 3.17. The system $\mathrm{RCA}_{0}^{\omega}+\mathrm{QF}-\mathrm{AC}^{1,1}$ proves $\mathrm{LIN} \leftrightarrow \mathrm{LIL}$.

Proof. Similar to the proof of Theorem 3.15] the reverse direction is immediate, while in case of $\neg\left(\exists^{2}\right)$ each principle is provable in $\operatorname{RCA}_{0}^{\omega}$ using the sub-cover consisting of all rationals. In case of $\left(\exists^{2}\right)$, let $\psi$ be as in LIL and consider $(\forall x \in \mathbb{R})(\exists y \in$ $\mathbb{R})\left(x \in J_{y}^{\psi}\right)$. Since the innermost formula is $\Sigma_{1}^{0}$, we may apply QF-AC ${ }^{1,1}$ to obtain $\Xi$ such that $(\forall x \in \mathbb{R})\left(x \in J_{\Xi(x)}^{\psi}\right)$. Since $\exists^{2}$ provides a functional that converts real numbers to a binary representation, we may assume that $\Xi$ is extensional on the reals. Now define $\Psi: I \rightarrow \mathbb{R}^{+}$by $\Psi(x):=\min (|x-\psi(\Xi(x))(1)|,|x-\psi(\Xi(x))(2)|)$, and

\footnotetext{
${ }^{5}$ The countable sub-cover in the Lindelöf lemma can be given by a sequence of reals generating the intervals (strong version), or just a sequence of intervals (weak version). The strong version implies QF-AC ${ }^{0,1}$ and hence is unprovable in $Z F$, while the weak version is provable in $Z_{2}^{\Omega}$.
} 
note that $I_{x}^{\Psi} \subseteq J_{\Xi(x)}^{\psi}$. Applying LIN, we obtain a countable sub-cover of $\cup_{x \in I} I_{x}^{\Psi}$, say generated by $\Phi^{0 \rightarrow 1}$, and $\cup_{i \in \mathbb{N}} I_{\Xi(\Phi(i))}^{\psi}$ is a countable sub-cover of $\cup_{x \in I} I_{x}^{\psi}$.

For completeness, we also mention the following corollary.

Corollary 3.18. $R_{C A}^{\omega}+$ QF-AC ${ }^{1,1}$ proves $\operatorname{LIL} \leftrightarrow[\operatorname{dim}(\mathbb{R}) \leq 1] \leftrightarrow \mathbb{R}$ is paracompact.

Proof. We only prove the equivalence between LIL and the paracompactness of $\mathbb{R}$. By Theorem 3.17, it suffices to prove LIN. In case $\neg\left(\exists^{2}\right)$, the latter is provable outright, as all $\mathbb{R} \rightarrow \mathbb{R}$-functions are continuous, and then the rationals provide a countable sub-cover for any open cover as in LIN. Similarly, paracompactness reduces to countable paracompactness, and the latter is provable in $\mathrm{RCA}_{0}^{\omega}$ by [51, II.7.2]. In case of $\left(\exists^{2}\right)$, the paracompactness of $\mathbb{R}$ (and hence $I$ with minor modification) implies HBT by Theorem 3.11, and the aforementioned result HBT $\leftrightarrow$ [WKL + LIL] over $\mathrm{RCA}_{0}^{\omega}+\mathrm{QF}-\mathrm{AC}^{1,1}$ finishes the forward direction. The reverse direction is straightforward as $\exists^{2}$ decides inequalities between reals, and hence can easily refine the countable sub-cover provided by LIN.

As it turns out, we can avoid the use of QF-AC ${ }^{1,1}$ as follows

Theorem 3.19. The system $\mathrm{RCA}_{0}^{\omega}+\left(\kappa_{0}^{3}\right)+$ IND proves $\left[\mathrm{LIN}+\mathrm{QF}-\mathrm{AC}^{0,1}\right] \leftrightarrow \mathrm{LIL}$.

Proof. For the forward implication, in case $\neg\left(\exists^{2}\right)$, the rationals provides a countable sub-cover, as all functions on the reals are continuous by [28, Prop. 3.7]. In case of $\left(\exists^{2}\right)$, fix $\psi: \mathbb{R} \rightarrow \mathbb{R}^{2}$ as in LIL and formulate a version of (3.5) as follows:

$$
(\forall x \in \mathbb{R})(\exists n \in \mathbb{N}) \underline{(\exists y \in \mathbb{R})\left(\left(x-\frac{1}{2^{n}}, x+\frac{1}{2^{n}}\right) \subseteq J_{y}^{\psi}\right),}
$$

The underlined formula is again decidable thanks to $\exists^{3}$, and QF-AC ${ }^{1,0}$ yields a functional $\Psi: \mathbb{R} \rightarrow \mathbb{R}^{+}$such that the canonical cover $\cup_{x \in \mathbb{R}} I_{x}^{\Psi}$ also covers $\mathbb{R}$. Applying LIN, we obtain a functional $\Phi^{0 \rightarrow 1}$ and the following version of (3.6):

$$
(\forall n \in \mathbb{N})\left(\exists v^{1^{*}}\right)(\forall i \leq n)\left(I_{\Phi(i)}^{\Psi} \subseteq I_{v(i)}^{\psi}\right) .
$$

Applying QF-AC ${ }^{0,1}$ to (3.8), we obtain LIL, and this direction is done.

For the reverse implication, note that LIL $\rightarrow$ QF-AC ${ }^{0,1}$ follows from 43 , Theorem 5.3], because the base theory $\mathrm{RCA}_{0}^{\omega}+\left(\kappa_{0}^{3}\right)$ allows us to generalise the class of covers, as discussed in [43, Remark 5.9]. With that, we are done.

We believe that the previous splitting 6 is proper. The following corollary to the theorem is proved in the same way.

Corollary 3.20. The system $\mathrm{RCA}_{0}^{\omega}+\left(\kappa_{0}^{3}\right)+$ IND proves

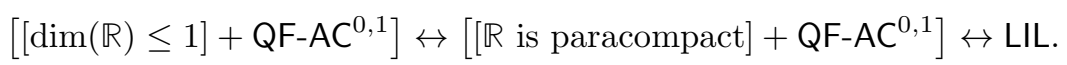

\footnotetext{
${ }^{6}$ In LIN, any $x \in \mathbb{R}$ is covered by $I_{x}^{\Psi}$, while in LIL any $x \in \mathbb{R}$ is covered by $J_{y}^{\psi}$ for some $y \in \mathbb{R}$. In the former case, we 'know' which interval covers the point, while in the latter case, we only know that it exists. We believe this (seemingly minor) difference determines whether one can obtain QF-AC 0,1 (like in the case of LIL) or not (in the case of LIN, we conjecture). Indeed, applying QF-AC ${ }^{1,0}$ to the conclusion of LIL, we obtain a functional that provides for any $x \in \mathbb{R}$, an interval $J_{y}^{\psi}$ covering $x$, i.e. LIL clearly exhibits 'axiom of choice' behaviour, while LIN does not.
} 
In conclusion, it is easy to see that the proofs of this section go through for the Lindelöf lemma for $\mathbb{R}$ based on any 'reasonable' notion of cover. Indeed, as long as the formulas ' $x \in U_{y}$ ' and ' $[a, b] \subseteq U_{x}$ ' for the new notion of cover $\cup_{x \in \mathbb{R}} U_{x}$ of $\mathbb{R}$ are decidable in $Z_{2}^{\Omega}$, the above proofs go through (assuming $\left(\kappa_{0}^{3}\right)$ ). Since $Z_{2}^{\Omega}$ can decide if $Y: \mathbb{R} \rightarrow\{0,1\}$ represents an open subset of $\mathbb{R}$ (using the textbook definition of open set), this notion of 'reasonable' again seems quite reasonable.

3.4.3. Partitions of unity. The notion of partition of unity was introduced in 1937 by Dieudonné in [13] and this notion is equivalent to paracompactness in a rather general setting by [17, Theorem 5.1.9]. We study partitions of unity in this section motivated as follows: on one hand, Simpson proves the existence of partitions of unity for complete separable spaces in the proof of [51, II.7.2], i.e. this notion has been studied in RM.

The definition of partition of unity is as follows in Munkres [37, p. 258]

Definition 3.21. Let $\left\{U_{\alpha}\right\}_{\alpha \in J}$ be an indexed open covering of $X$. An indexed family of continuous functions $\phi_{\alpha}: X \rightarrow[0,1]$ is said to be a partition of unity on $X$, dominated $\sqrt{7}$ by $\left\{U_{\alpha}\right\}$, if:

(1) $\operatorname{support}\left(\phi_{\alpha}\right) \subset U_{\alpha}$ for each $\alpha \in J$.

(2) The indexed family $\left\{\operatorname{support}\left(\phi_{\alpha}\right)\right\}_{\alpha \in J}$ is locally finite.

(3) $\sum_{\alpha \in J} \phi_{\alpha}(x)=1$ for each $x \in X$.

where support $(f)$ is the closure of the open set $\{x \in X: f(x) \neq 0\}$.

Note that the second item implies that the sum in the third one makes sense. With this definition in place, $\operatorname{PUNI}(I)$ is the statement that for any cover generated by $\psi: I \rightarrow \mathbb{R}$, there is a partition of unity $\phi: I \times I \rightarrow \mathbb{R}$ of $I$ dominated by $\cup_{x \in I} I_{x}^{\psi}$.

Theorem 3.22. The system $\mathrm{RCA}_{0}^{\omega}+\left(\kappa_{0}^{3}\right)+\mathrm{IND}$ proves $[\mathrm{WKL}+\mathrm{PUNI}(I)] \leftrightarrow \mathrm{HBT}$.

Proof. In case of $\neg\left(\exists^{2}\right)$, the equivalence is easy: all $\mathbb{R} \rightarrow \mathbb{R}$-functions are continuous and $\operatorname{PUNI}(I)$ is provable as in the proof of [51, II.7.2], while HBT follows from WKL as in the proof of Corollary 3.6. In case of $\left(\exists^{2}\right)$, the reverse implication is also straightforward: the finite sub-cover provided by HBT is readily refined, and the existence of a partition of unity for a finite cover follows from [51, II.7.1].

Finally, for the forward direction assuming $\left(\exists^{2}\right)$, let $\psi: I \rightarrow \mathbb{R}$ be as in HBT and obtain $\phi: I^{2} \rightarrow \mathbb{R}$ as in $\operatorname{PUNI}(I)$, i.e. for $V_{x}:=\operatorname{support}(\phi(x, \cdot))$, the open cover $\cup_{x \in I} V_{x}$ of $I$ is locally finite and satisfies $V_{x} \subset I_{x}^{\psi}$. Now consider:

$$
(\forall x \in I)(\exists n \in \mathbb{N}) \underline{(\exists y \in I)\left(\left(x-\frac{1}{2^{n}}, x+\frac{1}{2^{n}}\right) \subseteq V_{y}\right)},
$$

Applying QF-AC ${ }^{1,0}$ to 3.10 , since $\exists^{3}$ is given, we obtain $\Psi: I \rightarrow \mathbb{R}^{+}$such that $\cup_{x \in I} I_{x}^{\Psi}$ covers $I$. Now repeat the proof of Theorem 3.11 for $\Psi$ in place of $\phi$, which yields $y_{1}, \ldots y_{k} \in I$ such $\cup_{i \leq k} I_{x}^{\Psi}$ is a finite sub-cover of $I$. Note that in the previous 'repeated proof', we do not need the function $\Xi$ (from the proof of Theorem 3.5), as $I_{x}^{\Psi}$ covers $x$ for any $x \in I$. The aforementioned finite sub-cover of $\cup_{x \in I} I_{x}^{\Psi}$ now yields a finite sub-cover of $\cup_{x \in I} I_{x}^{\psi}$ using IND in the same way as for Theorem 3.16 .

Corollary 3.23. The system $\mathrm{RCA}_{0}^{\omega}+\mathrm{IND}+\left(\kappa_{0}^{3}\right)+\mathrm{PUNI}(I)$ proves $\mathrm{HBU} \leftrightarrow \mathrm{HBT}$.

\footnotetext{
7Munkres uses 'dominated by' in [37] instead of Engelking's 'subordinate to' in [17.
} 
Note that previous base theory in the corollary (and hence the theorem) is conservative over Peano arithmetic by [28, Prop. 3.12] and the proof of [51, II.7.2].

Next, we obtain a theorem that brings together a number of different strands from this paper, including effective paracompactness, first discussed at the end of Section 3.3. In the context of $\operatorname{RCA}_{0}^{\omega}$, we say that ' $\phi: \mathbb{R} \rightarrow \mathbb{R}$ is an effective refinement of $\psi: \mathbb{R} \rightarrow \mathbb{R}^{\prime}$ if $(\exists \xi: \mathbb{R} \rightarrow \mathbb{R})(\forall x \in \mathbb{R})\left(I_{x}^{\phi} \subseteq I_{\xi(x)}^{\psi}\right)$. Effective paracompactness expresses the existence of an effective refinement for any open cover. Moreover, $\operatorname{PUNI}(\mathbb{R})$ is the statement that for any cover generated by $\psi: \mathbb{R} \rightarrow \mathbb{R}^{2}$, there is a partition of unity $\phi: \mathbb{R}^{2} \rightarrow \mathbb{R}^{2}$ dominated by $\cup_{x \in I} J_{x}^{\psi}$.

Theorem 3.24. The system $\mathrm{RCA}_{0}^{\omega}+\left(\kappa_{0}^{3}\right)+$ IND proves the following

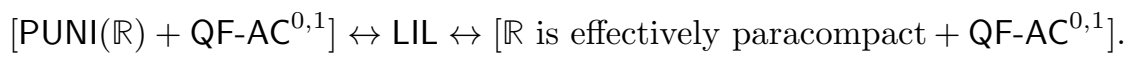

Proof. We first prove the first equivalence. In case of $\neg\left(\exists^{2}\right)$, the equivalence is easy: all $\mathbb{R} \rightarrow \mathbb{R}$-functions are continuous and $\operatorname{PUNI}(\mathbb{R})$ is provable as in the proof of $[51$, II.7.2], while LIL follows by taking the countable sub-cover given by the rationals. In case of $\left(\exists^{2}\right)$, the reverse implication is also straightforward: the countable subcover provided by LIL is readily refined, and the existence of a partition of unity for a countable cover follows from [51, II.7.1].

Finally, for the forward direction assuming $\left(\exists^{2}\right)$, let $\psi: \mathbb{R} \rightarrow \mathbb{R}^{2}$ be as in LIL and obtain $\phi: \mathbb{R}^{2} \rightarrow \mathbb{R}^{2}$ as in $\operatorname{PUNI}(\mathbb{R})$, i.e. for $V_{x}:=\operatorname{support}(\phi(x, \cdot))$, the open cover $\cup_{x \in \mathbb{R}} V_{x}$ of $\mathbb{R}$ is locally finite and satisfies $V_{x} \subset I_{x}^{\psi}$. Now consider:

$$
(\forall x \in \mathbb{R})(\exists n \in \mathbb{N}) \underline{(\exists y \in \mathbb{R})\left(\left(x-\frac{1}{2^{n}}, x+\frac{1}{2^{n}}\right) \subseteq U_{y}\right),}
$$

Applying QF-AC $C^{1,0}$ to (3.11), since $\exists^{3}$ is given, we obtain $\Psi: \mathbb{R} \rightarrow \mathbb{R}^{+}$such that $\cup_{x \in \mathbb{R}} I_{x}^{\Psi}$ covers $\mathbb{R}$. Now repeat the proof of Theorem 3.11 for $\Psi$ in place of $\phi$ and $\mathbb{R}$ instead of $I$. Then instead of (3.1), we make use of the following:

$$
(\forall x \in \mathbb{R})(\exists n \in \mathbb{N})\left(\zeta(n) \geq_{\mathbb{R}}|x|\right) .
$$

Note that in this 'repeated proof', we do not need the choice function $\Xi$ (from the proof of Theorem 3.5), as $I_{x}^{\Psi}$ covers $x$ for any $x \in I$. Applying QF-AC ${ }^{1,0}$ to (3.12), we obtain $\Phi^{0 \rightarrow 1}$ such that $\cup_{n \in \mathbb{N}} I_{\Phi(n)}^{\Psi}$ is a countable sub-cover of the canonical cover generated by $\Psi$. This countable sub-cover of $\cup_{x \in I} I_{x}^{\Psi}$ now yields a countable subcover of $\cup_{x \in I} I_{x}^{\psi}$ using IND and QF-AC ${ }^{0,1}$ in the same way as for Theorem 3.19.

3.5. A better base theory. The results in the previous section are not completely satisfactory: while the Axiom of Choice is avoided (as much as possible), the use of $\left(\kappa_{0}^{3}\right)$ amounts to little more than a trick. In this section, we develop a better approach based on the neighbourhood function principle NFP from [56, which is used in [42, §3] to derive e.g. HBU. As will become clear, the NFP principle gives rise to an elegant base theory for the results in the previous section.

We now introduce a fragment of NFP that is a generalisation of QF-AC $C^{1,0}$ to the following formula class. We always assume that $Y$ has type $(1 \times 0) \rightarrow 0$.

Definition 3.25. [C-formula] A basic $C$-formula has the form $\left(\exists f \in 2^{\mathbb{N}}\right)\left(Y\left(f, n^{0}\right)=\right.$ 0 ). A (general) $C$-formula is obtained from basic $C$-formulas via $\wedge, \vee, \rightarrow$, and $\neg$.

Note that $C$-formulas can have parameters besides the number variable, but quantifiers are restricted to $C$ and do not alternate inside a basic $C$-formula. The following axiom was first studied in [44] as an extension of the results in [48. 
Definition 3.26. $[C$-NFP $]$ For any $C$-formula $A\left(\sigma^{0^{*}}\right)$, we have

$$
\left(\forall f^{1}\right)\left(\exists n^{0}\right) A(\bar{f} n) \rightarrow\left(\exists \Phi^{2}\right)\left(\forall f^{1}\right) A(\bar{f} \Phi(f)) .
$$

By the below results, $C$-NFP 0 yields a good base theory for the RM of topology. There are however other (more conceptual) reasons for adopting this axiom. For instance, we prove $\left[\mathrm{HBU}+C\right.$-NFP 0 ] $\leftrightarrow C$-WKL over $\mathrm{RCA}_{0}^{\omega}+$ IND in [44, §4], where $C$-WKL is WKL with tree-elementhood ' $\sigma \in T$ ' given by a $C$-formula. Kohlenbach has studied similar generalisations of WKL in [29].

We now have the following corollary to Theorem 3.16 .

Corollary 3.27. The system $\mathrm{RCA}_{0}^{\omega}+\mathrm{IND}+C$-NFP proves $\mathrm{HBU} \leftrightarrow \mathrm{HBT}$

Proof. We show that $C$-NFP 0 applies to (3.5) and the rest of the proof is identical. Now, it is instructive to (equivalently) write (3.5) as follows:

$$
(\forall x \in I)(\exists n \in \mathbb{N})(\exists y \in I)\left(\left([x](n+1)-\frac{1}{2^{n+1}},[x](n+1)+\frac{1}{2^{n+1}}\right) \subseteq I_{y}^{\psi}\right),
$$

where $[x](n)$ is the $n$-th approximation of $x$. One can then write the underlined formula in (3.13) as $A(\bar{x} n)$ with only minor abuse of notation. Note that we need $\exists^{2}$ to convert $A(\bar{x} n)$ into a $C$-formula (using a binary representation for $y$ ).

We now have the following corollary to Theorem 3.19.

Corollary 3.28. The system $\mathrm{RCA}_{0}^{\omega}+\mathrm{IND}+C$-NFP $\mathrm{N}_{0}$ proves $\left[\mathrm{LIN}+\mathrm{QF}-\mathrm{AC}^{0,1}\right] \leftrightarrow \mathrm{LIL}$.

Proof. Similar to the proof of Corollary 3.27, replace (3.7) by

$$
(\forall x \in \mathbb{R})(\exists n \in \mathbb{N})(\exists y \in \mathbb{R})\left(\left([x](n+1)-\frac{1}{2^{n+1}},[x](n+1)+\frac{1}{2^{n+1}}\right) \subseteq J_{y}^{\psi}\right),
$$

and note that $C$-NFP 0 applies.

In light of the previous corollaries, it is clear one can similarly replace $\left(\kappa_{0}^{3}\right)$ by $C$-NFP ${ }_{0}$ in Theorems 3.22 and 3.24 (and corollaries) by considering modifications of (3.10) and (3.11) similar to (3.13) and (3.14).

By the above, $C$-NFP 0 seems to be an acceptable/usefull generalisation of QF-AC ${ }^{1,0}$ and should be adopted as part of the base theory as well. We invite the reader to ponder a similar generalisation of $\Delta_{1}^{0}$-comprehension. The answer is given in [48].

\section{Conclusion}

We have studied the higher-order RM of topology, the notions of dimension and paracompactness in particular. Basic theorems regarding the latter turn out to be equivalent to the Heine-Borel theorem for uncountable covers, i.e. the former are extremely hard to prove (in terms of comprehension axioms). A number of nice splittings was obtained, and we have shown that these results do not depend on the exact definition of cover, even in the absence of the axiom of choice. In this section, we discuss the foundational implications of our results, esp. as they pertain to the Gödel hierarchy. Now, the latter is a collection of logical systems ordered via consistency strength. This hierarchy is claimed to capture most systems that are natural or have foundational import, as follows.

It is striking that a great many foundational theories are linearly ordered by $<$. Of course it is possible to construct pairs of artificial theories which are incomparable under $<$. However, this is not the case for the "natural" or non-artificial theories which are usually regarded as significant in the foundations of mathematics. ([50]) 
Burgess and Koellner corroborate this claim in [7, §1.5] and [27, §1.1]. The Gödel hierarchy is a central object of study in mathematical logic, as e.g. argued by Simpson in [50, p. 112] or Burgess in [7, p. 40]. Precursors to the Gödel hierarchy may be found in the work of Wang ([59]) and Bernays (see [5, [6]). Friedman ([20]) studies the linear nature of the Gödel hierarchy in detail. Moreover, the Gödel hierarchy exhibits some remarkable robustness: we can perform the following modifications and the hierarchy remains largely unchanged:

(i) Instead of the consistency strength ordering, we can order via inclusion: Simpson claims that inclusion and consistency strength yield the same Gödel hierarchy as depicted in [50, Table 1]. Some exceptional (seminatural) statement 9 do fall outside of the inclusion-based Gödel hierarchy.

(ii) We can replace the systems with their higher-order (eponymous but for the ' $\omega$ ') counterparts. The higher-order systems are generally conservative over their second-order counterpart for (large parts of) $\mathrm{L}_{2}$. Hunter's dissertation contains a number of such general results ([23, Ch. 2]).

Now, if one accepts the modifications (inclusion ordering and higher types) described in the previous two items, then an obvious question is where basic topological theorems pertaining to e.g. dimension and paracompactness fit within the Gödel hierarchy. As depicted in Figure 1, the aforementioned theorems yield a branch that is completely independent of the medium range of the Gödel hierarchy (with the latter based on inclusion). The same 'independence' holds for basic properties of the gauge integral, including many covering lemmas (see [42]), as well as for so-called uniform theorems (see [43]) in which the objects claimed to exist depend on few of the parameters of the theorem. Some remarks on the technical details concerning Figure 1 are as follows.

Remark 4.1. First of all, we use a non-essential modification of the Gödel hierarchy, namely involving systems of higher-order arithmetic, like e.g. ACA $_{0}^{\omega}$ instead of $\mathrm{ACA}_{0}$; these systems are (at least) $\Pi_{2}^{1}$-conservative over the associated second-order system (see e.g. [46, Theorem 2.2]).

Secondly, $\mathrm{Z}_{2}^{\Omega}$ is placed between the medium and strong range, as the combination of the recursor $R_{2}$ from Gödel's $T$ and $\exists^{3}$ yields a system stronger than $Z_{2}^{\Omega}$. The system $\Pi_{k}^{1}-\mathrm{CA}_{0}^{\omega}$ does not change in the same way.

Thirdly, while HBT clearly implies WKL, the paracompactness of the unit interval does not (by the ECF-translation); this is symbolised by the dashed line.

Fourth, while HBT and similar statements are hard to prove (in terms of comprehension axioms), these theorems (must) have weak first-order strength in light of their provability in intuitionistic topology (see e.g. [54,57]).

The previous remark also establishes that the systems with superscript ' $\omega$ ' deserve to be called the higher-order counterparts of the corresponding second-order systems, while $Z_{2}^{\Omega}$ does not fall into the same category.

\footnotetext{
${ }^{8}$ Simpson mentions in [50] the caveat that e.g. PRA and $\mathrm{WKL}_{0}$ have the same first-order strength, but the latter is strictly stronger than the former.

${ }^{9}$ There are some examples (predating HBU and [42] that fall outside of the Gödel hierarchy based on inclusion, like special cases of Ramsey's theorem and the axiom of determinacy from set theory $(22,32])$. These are far less natural than e.g. Heine-Borel compactness, in our opinion.
} 


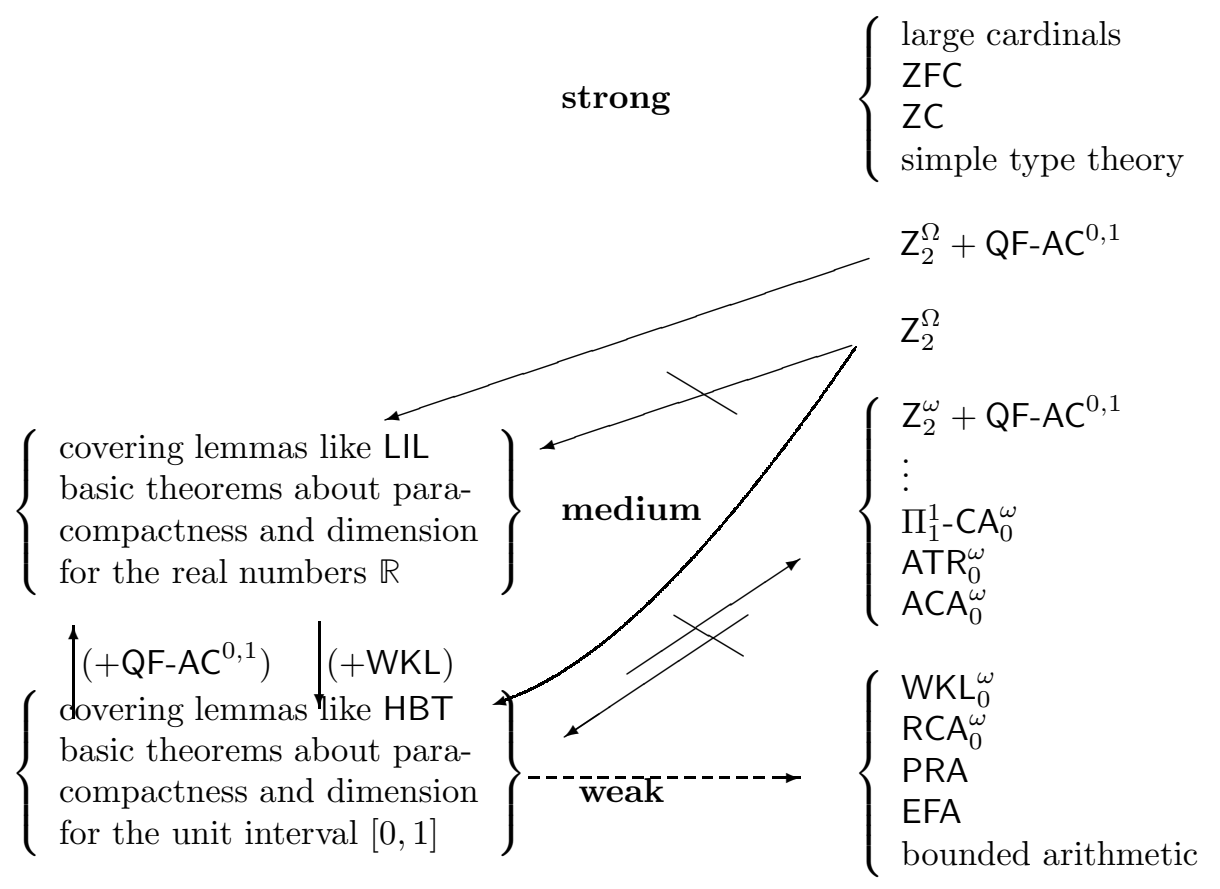

Figure 1. The Gödel hierarchy with a side-branch for the medium range

In light of the equivalences involving the gauge integral, uniform theorems, and the Cousin lemma (and hence HBT) from [42, 43], one is tempted to see a serious challenge to the 'Big Five' classification from RM, the linear nature of the Gödel hierarchy, as well as Feferman's claim that the mathematics necessary for the development of physics can be formalised in relatively weak logical systems (see [42, p. 24]). While such an 'anti-establishment' view is indeed tempting, a more enlightened interpretation of the aforementioned equivalences can be found in 48. In a nuthshell, the second-order part of the Gödel hierarchy (including equivalences) is merely the result of applying the ECF-translation to a carefully formulated higher-order hierarchy; this translation maps equivalences to equivalences.

Regarding future work, the following two topics come to mind. Firstly, there are a number of notions weaker than paracompactness, and it is an interesting question if there are natural such notions that yield equivalences with HBT or weaker theorems. Secondly, in light of Remark 3.13, it seems interesting to study metrisation theorems in higher-order RM. We expect that such theorems go far beyond $\Pi_{2}^{1}-\mathrm{CA}_{0}$, which features in the second-order RM of topology.

Acknowledgement 4.2. Our research was supported by the John Templeton Foundation, the Alexander von Humboldt Foundation, LMU Munich (via the Excellence Initiative and the Center for Advanced Studies of LMU), and the University of Oslo. We express our gratitude towards these institutions. We thank Dag Normann for his valuable advice. Opinions expressed in this paper do not reflect those of the John Templeton Foundation. 


\section{REFERENCES}

[1] Encyclopaedia of mathematics. Vol. 2. C, Kluwer Academic Publishers, Dordrecht, 1988. Translated from the Russian; Translation edited by M. Hazewinkel.

[2] Jeremy Avigad and Solomon Feferman, Gödel's functional ("Dialectica") interpretation, Handbook of proof theory, Stud. Logic Found. Math., vol. 137, 1998, pp. 337-405.

[3] Robert Bartle, A modern theory of integration., Grad. Stud. Math. 32 (2001), xiv + 458.

[4] Michael J. Beeson, Foundations of constructive mathematics, Ergebnisse der Mathematik und ihrer Grenzgebiete, vol. 6, Springer, 1985. Metamathematical studies.

[5] Paul Bernays, Sur le Platonisme Dans les Mathématiques, L'Enseignement Mathématique 34 (1935), 52-69.

[6] Paul Benacerraf and Hilary Putnam, Philosophy of Mathematics: Selected Readings, 2nd ed., Cambridge University Press, 1984.

[7] John P. Burgess, Fixing Frege, Princeton Monographs in Philosophy, Princeton University Press, 2005.

[8] Frank E. Burk, A garden of integrals, The Dolciani Mathematical Expositions, vol. 31, Mathematical Association of America, Washington, DC, 2007.

[9] Pierre Cousin, Sur les fonctions de $n$ variables complexes, Acta Math. 19 (1895), no. 1, 1-61.

[10] A. Denjoy, Une extension de l'intégrale de M. Lebesgue., C. R. Acad. Sci., Paris 154 (1912), 859-862.

[11] Jean Dieudonné, A history of algebraic and differential topology 1900-1960, Birkhäuser Boston, 2009.

[12] Une généralisation des espaces compacts, J. Math. Pures Appl. (9) 23 (1944), 65-76 (French).

[13] _ Sur les fonctions continues numériques definies dans un produit de deux espaces compacts, C. R. Acad. Sci., Paris 205 (1937), 593-595 (French).

[14] Lejeune P. G. Dirichlet, Sur la convergence des séries trigonométriques qui servent à représenter une fonction arbitraire entre des limites données, arXiv (2008). https://arxiv.org/abs/0806.1294

[15] Damir D. Dzhafarov, Reverse Mathematics Zoo. http://rmzoo.uconn.edu/

[16] Ryszard Engelking, Dimension theory, 1978. North-Holland Mathematical Library, 19.

[17] — General topology, 2nd ed., Sigma Series in Pure Mathematics, vol. 6, Heldermann Verlag, Berlin, 1989.

[18] Harvey Friedman, Some systems of second order arithmetic and their use, Proceedings of the International Congress of Mathematicians (Vancouver, B. C., 1974), Vol. 1, 1975, pp. 235-242.

[19] — Systems of second order arithmetic with restricted induction, I \& II (Abstracts), Journal of Symbolic Logic 41 (1976), 557-559.

[20] - Interpretations, According to Tarski, Interpretations of Set Theory in Discrete Mathematics and Informal Thinking, The Nineteenth Annual Tarski Lectures, http://u.osu.edu/friedman.8/files/2014/01/Tarski1052407-13do0b2.pdf 1 (2007), pp. 42 .

[21] C. Good, I. J. Tree, and W. S. Watson, On Stone's theorem and the axiom of choice, Proc. Amer. Math. Soc. 126 (1998), no. 4, 1211-1218.

[22] Denis R. Hirschfeldt, Slicing the truth, Lecture Notes Series, Institute for Mathematical Sciences, National University of Singapore, vol. 28, World Scientific Publishing, 2015.

[23] James Hunter, Higher-order reverse topology, ProQuest LLC, Ann Arbor, MI, 2008. Thesis (Ph.D.)-The University of Wisconsin - Madison.

[24] Kyriakos Keremedis, Disasters in topology without the axiom of choice, Arch. Math. Logic 40 (2001), no. 8.

[25] John L. Kelley, General topology, Springer-Verlag, 1975. Reprint of the 1955 edition; Graduate Texts in Mathematics, No. 27.

[26] Israel Kleiner, Excursions in the history of mathematics, Birkhäuser/Springer, New York, 2012.

[27] Peter Koellner, Large Cardinals and Determinacy, The Stanford Encyclopedia of Philosophy, 2014. https://plato.stanford.edu/archives/spr2014/entries/large-cardinals-determinacy/

[28] Ulrich Kohlenbach, Higher order reverse mathematics, Reverse mathematics 2001, Lect. Notes Log., vol. 21, ASL, 2005, pp. 281-295. 
[29] - Foundational and mathematical uses of higher types, Reflections on the foundations of mathematics (Stanford, CA, 1998), Lect. Notes Log., vol. 15, ASL, 2002, pp. 92-116.

[30] Ernst Lindelöf, Sur Quelques Points De La Théorie Des Ensembles, Comptes Rendus (1903), 697-700.

[31] Fyodor A. Medvedev, Scenes from the history of real functions, Science Networks. Historical Studies, vol. 7, Birkhäuser Verlag, Basel, 1991.

[32] A. Montalbán and Richard A. Shore, The limits of determinacy in second-order arithmetic, Proc. Lond. Math. Soc. (3) 104 (2012), no. 2, 223-252.

[33] A. Montalbán, Open questions in reverse mathematics, Bull. Symb. Logic 17 (2011), 431-454.

[34] P. Muldowney, A general theory of integration in function spaces, including Wiener and Feynman integration, Pitman Research Notes in Mathematics Series, vol. 153, Longman Scientific \& Technical, Harlow; John Wiley \& Sons, Inc., New York, 1987.

[35] - The infinite dimensional Henstock integral and problems of Black-Scholes expectation, J. Appl. Anal. 8 (2002), no. 1, 1-21.

[36] Pat Muldowney, A modern theory of random variation, John Wiley \& Sons, 2012. With applications in stochastic calculus, financial mathematics, and Feynman integration.

[37] James R. Munkres, Topology, Prentice-Hall, 2000, 2nd edition.

[38] Carl Mummert and Stephen G. Simpson, Reverse mathematics and $\Pi_{2}^{1}$ comprehension, Bull. Symbolic Logic 11 (2005), no. 4, 526-533.

[39] Carl Mummert, On the reverse mathematics of general topology, ProQuest LLC, Ann Arbor, MI, 2005. Thesis (Ph.D.)-The Pennsylvania State University.

[40] - Reverse mathematics of MF spaces, J. Math. Log. 6 (2006), no. 2, 203-232.

[41] Dag Normann and Sam Sanders, Nonstandard Analysis, Computability Theory, and their connections, To appear in Journal of Symbolic Logic, arXiv: https://arxiv.org/abs/1702.06556 (2017).

[42] - On the mathematical and foundational significance of the uncountable, To appear in Journal of Mathematical Logic, arXiv: https://arxiv.org/abs/1711.08939 (2018).

[43] _ Pincherle's theorem in computability theory and Reverse Mathematics, To appear in Annals of Pure and Applied Logic, arxiv: https://arxiv.org/abs/1808.09783 (2020).

[44] _ Open sets in computability theory and Reverse Mathematics, Submitted, arxiv: https://arxiv.org/abs/1910.02489 (2019).

[45] Salvatore Pincherle, Sopra alcuni sviluppi in serie per funzioni analitiche (1882), Opere Scelte, I, Roma (1954), 64-91.

[46] Nobuyuki Sakamoto and Takeshi Yamazaki, Uniform versions of some axioms of second order arithmetic, MLQ Math. Log. Q. 50 (2004), no. 6, 587-593.

[47] Sam Sanders, Splittings and disjunctions in Reverse Mathematics, To appear in Notre Dame Journal for Formal Logic, https://arxiv.org/abs/1805.11342 (2018), pp. 20.

[48] - Plato and the Foundations of Mathematics, Submitted, arxiv: https://arxiv.org/abs/1908.05676 (2019), pp. 40.

[49] Stephen G. Simpson (ed.), Reverse mathematics 2001, Lecture Notes in Logic, vol. 21, ASL, La Jolla, CA, 2005.

[50] Stephen G. Simpson, The Gödel hierarchy and reverse mathematics., Kurt Gödel. Essays for his centennial, 2010, pp. 109-127.

[51] Stephen G. Simpson, Subsystems of second order arithmetic, 2nd ed., Perspectives in Logic, CUP, 2009.

[52] John Stillwell, Reverse mathematics, proofs from the inside out, Princeton Univ. Press, 2018.

[53] A. H. Stone, Paracompactness and product spaces, Bull. Amer. Math. Soc. 54 (1948), 977982.

[54] Anne Sjerp Troelstra, Intuitionistic general topology, PhD thesis, University of Amsterdam, 1966.

[55] - Metamathematical investigation of intuitionistic arithmetic and analysis, Springer Berlin, 1973. Lecture Notes in Mathematics, Vol. 344.

[56] Anne Sjerp Troelstra and Dirk van Dalen, Constructivism in mathematics. Vol. I, Studies in Logic and the Foundations of Mathematics, vol. 121, North-Holland, 1988.

[57] Frank Waaldijk, Modern intuitionistic topology, PhD thesis, University of Nijmegen, 1996.

[58] W. Stephen Watson, Pseudocompact metacompact spaces are compact, Proc. Amer. Math. Soc. 81 (1981), no. 1, 151-152.

[59] Hao Wang, Eighty years of foundational studies, Dialectica 12 (1958), 466-497. 
[60] K. Weierstraß, Ausgewählte Kapitel aus der Funktionenlehre, Teubner-Archiv zur Mathematik [Teubner Archive on Mathematics], vol. 9, BSB B. G. Teubner Verlagsgesellschaft, Leipzig, 1988. 